\title{
ERRATUM
}

\section{A silicon-based surface code quantum computer}

Joe O'Gorman, Naomi H Nickerson, Philipp Ross, John JL Morton and Simon C Benjamin

npj Quantum Information (2016) 2, 16014; doi:10.1038/npjqi.2016.14; published online 12 April 2016

Correction to: npj Quantum Information (2016) 2, 15019; doi:10.1038/npjqi.2015.19; published online 2 February 2016

The original version of this article contained errors.

In the Introduction:

The GitHub online link was incorrectly quoted and should appear as: "/naominickerson/fault_tolerance_simulations/ releases"

In the Results:

Page 2 , column 1, line 23 , the whole sentence should read:

'It is important that $\mathrm{d} \ll \mathrm{D}$, in order that any interactions between the in-plane spins are relatively weak'.

Page 2 , column 2 , line 30 , the whole sentence should read:
'Therefore, the Hamiltonian of interest describes two $S=1 / 2$ spins, each in a static $B$ field in the $Z$ direction and experiencing $a$ dipole-dipole interaction...'

Page 2 column 2, line 42, should read:

'... where the expressions discard irrelevant global phases, 1 is the...'

Page 4 column 1, line 6 , should read:

'...simple phase shifts induced by the 'deactivated' probe can either be...'

Page 5, figure 4, note a should read:

'Private communication with the author of [28]'

The errors have been corrected in the PDF and HTML versions of the article. 